TRANSACTIONS OF THE

AMERICAN MATHEMATICAL SOCIETY

Volume 360, Number 6, June 2008, Pages 3249-3261

S 0002-9947(08)04358-4

Article electronically published on January 30, 2008

\title{
KNOT ADJACENCY AND FIBERING
}

\author{
EFSTRATIA KALFAGIANNI AND XIAO-SONG LIN
}

\begin{abstract}
It is known that the Alexander polynomial detects fibered knots and 3-manifolds that fiber over the circle. In this note, we show that when the Alexander polynomial becomes inconclusive, the notion of knot adjacency can be used to obtain obstructions to the fibering of knots and of 3-manifolds. As an application, given a fibered knot $K^{\prime}$, we construct infinitely many nonfibered knots that share the same Alexander module with $K^{\prime}$. Our construction also provides, for every $n \in N$, examples of irreducible 3-manifolds that cannot be distinguished by the Cochran-Melvin finite type invariants of order $\leq n$.
\end{abstract}

\section{INTRODUCTION}

The problem of detecting fiberedness (or non-fiberedness) of knots has been studied considerably from both the algebraic and the geometric topology viewpoint. The classical Alexander polynomial of a fibered knot is known to be monic and this provides an effective criterion for detecting fibered knots. The converse is not in general true, although it holds for several special classes of knots including alternating knots and knots up to ten crossings. Non-commutative generalizations of the Alexander polynomial, such as the higher order Alexander polynomials defined in $[\mathrm{C}]$ and suitable versions of the twisted Alexander polynomials, are known to detect non-fibered knots with the monic Alexander polynomial ([GKM, Ch, FKi]). A geometric procedure to detect fibered knots was developed by Gabai in [Ga2].

The purpose of this paper is to introduce a new criterion for detecting non-fibered knots when the Alexander polynomial fails, and to present several applications of this criterion. Our approach combines both the algebraic and the geometric point of view. To describe our results, we recall that the knot $K$ is said to be $n$-adjacent to another knot $K^{\prime}$, if $K$ admits a projection containing $n$ crossings such that changing any $0<m \leq n$ of them yields a projection of $K^{\prime}$. The notion of knot adjacency was studied in [KL1] and the theory was further developed in [KL], [K1]. In particular, in [K1] we showed that high degree adjacency $(n>1)$ to fibered knots imposes strong restrictions on the knot genus. In this paper, we explore the role of knot adjacency as an obstruction to fiberedness. We show that when the Alexander polynomial provides inconclusive evidence, high degree knot adjacencies obstruct knots to be fibered. More precisely, we have the following.

Received by the editors July 15, 2005 and, in revised form, June 27, 2006.

2000 Mathematics Subject Classification. Primary 57M25, 57M27, 57M50.

Key words and phrases. Alexander polynomial, knot adjacency, fibered knots and 3-manifolds, finite type invariants, symplectic structures.

The research of the authors was partially supported by the NSF.

Xiao-Song Lin passed away on January 14, 2007.

(C)2008 American Mathematical Society Reverts to public domain 28 years from publication 
Theorem 1.1. Let $K, K^{\prime}$ be distinct knots with equal Alexander polynomials. Suppose that $K^{\prime}$ is fibered. If $K$ is n-adjacent to $K^{\prime}$ for some $n>1$, then $K$ is not fibered. Furthermore, the 3-manifold $K(0)$ obtained by 0-Dehn surgery of $S^{3}$ along $K$ does not admit a fibration over $S^{1}$.

Since it is known that all knots up to ten crossings that have monic Alexander polynomial are fibered ([Ga2]), the knots that are detected by Theorem 1.1 have large crossing numbers. In Section 2 we give a systematic procedure that constructs vast families of such knots. In particular, given a fibered knot $K^{\prime}$, we construct infinitely many non-fibered knots that share the same Alexander module with $K^{\prime}$. To state our results, for a knot $K$, let $\Delta_{K}(t)$ denote the Alexander polynomial of $K$, let $\delta(K)$ denote the degree of $\Delta_{K}(t)$ and let $g(K)$ denote the genus of $K$. It is well known that if a knot $K$ is fibered, then $m(K):=2 g(K)-\delta(K)=0$.

Theorem 1.2. Given a fibered knot $K^{\prime}$ there exist infinitely many knots $\left\{K_{n}\right\}_{n \in \mathbf{N}}$, such that:

(a) $K_{n}$ and $K^{\prime}$ have the same Alexander module.

(b) $g\left(K_{n}\right)>g\left(K^{\prime}\right)$ and $\frac{n+3}{6} \leq g\left(K_{n}\right)$.

(c) $m\left(K_{n}\right)>0$ and $\lim _{n \rightarrow \infty} m\left(K_{n}\right)=\infty$. In particular, $K_{n}$ is not fibered.

(d) $K_{n}(0)$ does not fiber over $S^{1}$.

Furthermore, if $K^{\prime}$ is a prime knot, then $K_{n}$ can be chosen to be prime.

In the course of the proof of Theorem 1.2, we show that all the knots $\left\{K_{n}\right\}_{n \in \mathbf{N}}$ share a common equivalence class of Seifert matrices with $K^{\prime}$. Thus, $K_{n}$ and $K$ cannot be distinguished by abelian invariants (e.g. torsion numbers, signature, Blanchfield linking forms). This gives a new proof of a slightly weaker version of the main result of $[\mathrm{Ch}]$.

As another application of Theorem 1.1, we construct families of 3-manifolds that cannot be distinguished by certain finite type invariants. For Z-homology 3spheres these invariants were defined by Ohtsuki in [O]. An extension of Ohtsuki's theory to arbitrary 3-manifolds was proposed by Cochran and Melvin in [CM]. There exist constructions that yield irreducible distinct homology 3-spheres with the same finite type invariants of bounded order $([\mathrm{K}])$. Here we give examples of irreducible 3-manifolds with non-trivial homology that cannot be distinguished by their finite type invariants in the sense of $[\mathrm{CM}]$. To state our results, for a knot $K$ and a number $s \in \mathbf{Q}$, let $K(s)$ denote the 3-manifold obtained by $s$-Dehn surgery of $S^{3}$ along $K$. We show that if $K, K^{\prime}$ are knots such that $K$ is $n$-adjacent to $K^{\prime}$, then, for every $s \in \mathbf{Q}, K(s)$ and $K^{\prime}(s)$ cannot be distinguished by any finite type invariant of order $<n$ (Proposition 4.3). Combining this with Theorem 1.1 we obtain the following:

Corollary 1.3. For every $n \in \mathbf{N}$, there exist closed, irreducible 3-manifolds $M, M^{\prime}$ such that:

(a) $M$ and $M^{\prime}$ have the homology type of $S^{2} \times S^{1}$.

(b) For every commutative ring $\mathcal{R}$ with unit, $M$ and $M^{\prime}$ have the same $\mathcal{R}$-valued Cochran-Melvin finite type invariants of order $<n$.

(c) Exactly one of $M, M^{\prime}$ fibers over $S^{1}$.

The examples of Corollary 1.3 are obtained by 0-Dehn surgery on knots $K \subset S^{3}$. If $0 \neq s:=\frac{a}{b}$, then $K(s)$ is a $\mathbf{Q}$-homology sphere. In particular, if $K$ is the unknot, 
then $K(s)$ is the Lens space $L(a, b)$ and thus $\pi_{1}(K(s))=\mathbf{Z}_{a}$. Combining our work with the Cyclic Surgery Theorem of Culler, Gordon, Luecke and Shalen ([CGLS]) we obtain the following:

Corollary 1.4. Given $n \in \mathbf{N}$, there exists a knot $K \subset S^{3}$ and an integer $r_{K}$ such that for every $s:=\frac{a}{b} \neq r_{K}, r_{K}+1$ we have:

(a) $\pi_{1}(K(s)) \neq \pi_{1}(L(a, b))$.

(b) For every commutative ring $\mathcal{R}$ with unit, $K(s)$ and $L(a, b)$ have the same $\mathcal{R}$-valued Cochran-Melvin finite type invariants of order $<n$.

The examples of Corollary 1.3 and Corollary 1.4 are the first known examples of irreducible, non-Z-homology spheres that are not distinguished by finite type invariants of bounded order.

The paper is organized as follows: In Sections 2 and 3 we state a fibering criterion in terms of knot adjacency and discuss its applications to detecting non-fibered knots and 3-manifolds. In Section 4 we begin by recalling from [CM] the definition of finite type invariants for arbitrary 3 -manifolds. Then, we apply our results from Sections 2 and 3 to construct examples of 3-manifolds with the same finite type invariants of bounded orders and to prove Corollaries 1.3 and 1.4. In Section 5, we give the proof of Theorem 1.3. Section 6 is a short appendix in which we discuss that, combined with work of Kronheimer, Theorem 1.2 can be used to detect the existence of symplectic structures on certain 4-manifolds.

\section{Adjacency to fibered knots and the Alexander polynomial}

Let $K$ be a knot in $\mathbf{S}^{3}$ and let $q \in \mathbf{Z}$. A generalized crossing of order $q$ on a projection of $K$ is a set $C$ of $|q|$ twist crossings on two strings that inherit opposite orientations from any orientation of $K$. If $K^{\prime}$ is obtained from $K$ by changing all the crossings in $C$ simultaneously, we will say that $K^{\prime}$ is obtained from $K$ by a generalized crossing change of order $q$. Note that if $|q|=1, K$ and $K_{1}$ differ by an ordinary crossing change while if $q=0$ we have $K=K^{\prime}$. A crossing disc corresponding to a generalized crossing $C$ of a knot $K$ is an embedded $\operatorname{disc} D \subset S^{3}$ such that $K$ intersects int $(D)$ twice, with a zero algebraic intersection number (once for each string of $K$ forming the crossing). The curve $\partial D$ is called a crossing circle corresponding to $C$. The crossing is called nugatory if $\partial D$ bounds a disc in the complement of $K$.

Definition 2.1. We will say that $K$ is $n$-adjacent to $K^{\prime}$, for some $n>0$, if $K$ admits a projection containing $n$ generalized crossings such that changing any $0<m \leq n$ of them yields a projection of $K^{\prime}$. We will write $K \stackrel{n}{\longrightarrow} K^{\prime}$.

For a knot $K$, let $g(K)$ denote its genus and let $\delta:=\delta(K)$ denote the degree of its Alexander polynomial $\Delta_{K}(t)=\sum_{i=0}^{\delta} a_{i} t^{i}$. We have the following theorem, that contains the first conclusion in Theorem 1.1.

Theorem 2.2. Let $K, K^{\prime}$ be knots such that $\delta(K)=\delta\left(K^{\prime}\right)$ and $K \stackrel{n}{\longrightarrow} K^{\prime}$, for some $n>1$. If $K^{\prime}$ is fibered, then either $K$ is isotopic to $K^{\prime}$ or we have $g(K)>g\left(K^{\prime}\right)$. Furthermore, in the later case, $K$ is neither fibered nor alternating.

Proof. Since $K^{\prime}$ is fibered, we have $\delta\left(K^{\prime}\right)=2 g\left(K^{\prime}\right)$. Thus we have

$$
\delta(K)=\delta\left(K^{\prime}\right)=2 g\left(K^{\prime}\right) .
$$


The assumption that $K \stackrel{n}{\longrightarrow} K^{\prime}$ allows as to apply the results of [KL1, K1]: Indeed, as it was shown in [K1], if $K$ is $n$-adjacent to a fibered knot $K^{\prime}$, for some $n>1$, then either $K$ is isotopic to $K^{\prime}$ or we have $g(K)>g\left(K^{\prime}\right)$. If $g(K)>g\left(K^{\prime}\right)$, then (1) implies that $m(K):=2 g(K)-\delta(K)>0$ and thus $K$ is not fibered or alternating.

By Corollary 8.19 of [Ga1], $K(0)$ fibers over $S^{1}$ precisely when $K$ is a fibered knot. Thus, the second conclusion of Theorem 1.1 holds. The next theorem and its proof provide vast collections of non-fibered knots with monic Alexander polynomials that are detected by Theorem 2.2.

Theorem 2.3. Given a fibered knot $K^{\prime}$ and $n>1$, there exists a knot $K$ with the following properties:

(a) $K \stackrel{n}{\longrightarrow} K^{\prime}$.

(b) $K$ and $K^{\prime}$ have the same Alexander module.

(c) $g(K)>g\left(K^{\prime}\right)$.

(d) If $K^{\prime}$ is a prime knot, then $K$ can be chosen to be prime.

Remark 2.4. Theorem 2.3 (a)-(c) remains true for $n=1$. To see this, let $J$ be a knot with trivial Alexander polynomial that can be unknotted by a single generalized crossing change (e.g. an untwisted Whitehead double of any knot). Then the connected sum $K:=J \# K^{\prime}$ has the properties (a)-(c) of Theorem 2.3.

The proof of Theorem 2.3 and that of Theorem 1.2 are given in section 5 . In the next two sections we present applications of Theorems 1.1 and 1.2.

\section{Obstructing Fibrations}

Let $\mathcal{K}_{1}$ denote the set of isotopy classes of knots with monic Alexander polynomial and recall that for $K \in \mathcal{K}_{1}$, we denote $m(K):=2 g(K)-\delta(K) \geq 0$, where $\delta(K)$ is the degree of the Alexander polynomial of $K$. For $K \in \mathcal{K}_{1}$, let $F_{K} \subset \mathcal{K}_{1}$ denote the set of isotopy classes of fibered knots, that are distinct from $K$ but share the same Alexander polynomial with $K$. As shown in [Mo], if $\delta(K)>2$, then $F_{K}$ is infinite. On the other hand, it is known that the only fibered knots of genus $\leq 1$ are the trefoils, the figure eight and the unknot. It is easy to see that all degree $\leq 2$ monic Alexander polynomials are realized by these knots. Thus, if $\delta(K) \leq 2$, then $F_{K}$ is finite. Fix $K \in \mathcal{K}_{1}$. For $K^{\prime} \in F_{K}$, let

$$
I\left(K, K^{\prime}\right):=\left\{n>1 \mid K \stackrel{n}{\longrightarrow} K^{\prime}\right\} .
$$

We define $a\left(K, K^{\prime}\right):=\max \left\{n \in I\left(K, K^{\prime}\right)\right\}$ if the set $I\left(K, K^{\prime}\right)$ is non-empty. Otherwise, we define $a\left(K, K^{\prime}\right):=0$. Finally, we define

$$
\alpha(K):=\max \left\{a\left(K, K^{\prime}\right), K^{\prime} \in F_{K}\right\} .
$$

The quantity $\alpha(K)$ is clearly an invariant of $K$. Roughly speaking, it measures the degree of adjacency of a knot with monic Alexander polynomial to fibered knots with the same polynomial. By definition, we have $\alpha(K) \in \mathbf{N} \cup\{\infty\}$; the following proposition shows that, in fact, $\alpha(K)<\infty$, for every $K \in \mathcal{K}_{1}$.

Proposition 3.1. The invariant $\alpha$ has the following properties:

(a) We have $0 \leq \alpha(K) \leq 6 g(K)-3$, for every $K \in \mathcal{K}_{1}$.

(b) If $m(K)=0$, then $\alpha(K)=0$. In particular, if $K$ is fibered, then $\alpha(K)=0$. 
Proof. Clearly we have $\alpha(K) \geq 0$. By definition, if $\alpha(K)>0$, then there is a fibered knot $K^{\prime} \neq K$ such that $\Delta_{K}(t)=\Delta_{K^{\prime}}(t)$ and $K \stackrel{n}{\longrightarrow} K^{\prime}$, for some $n>1$. By Theorem 2.2, we must have $g(K)>g\left(K^{\prime}\right)$, and Theorem 1.3 of [KL1] applies to conclude that $n \leq 6 g(K)-3$. Thus part (a) is proved. To see part (b) suppose that $m(K):=2 g(K)-\delta(K)=0$ and that $\alpha(K)>0$. Then, by definition, there is a fibered knot $K^{\prime} \neq K$ such that $\delta\left(K^{\prime}\right)=\delta(K)$ and $K \stackrel{n}{\longrightarrow} K^{\prime}$, for some $n>1$. Since $K^{\prime}$ is fibered we have $\delta\left(K^{\prime}\right)=2 g\left(K^{\prime}\right)$. But since $\delta(K)=2 g(K)$, we conclude that $g(K)=g\left(K^{\prime}\right)$. But this is impossible, since by Theorem 2.2 we must have $g(K)>g\left(K^{\prime}\right)$.

The proof of the next corollary uses $\alpha(K)$ to produce infinitely many non-fibered knots with a given monic Alexander polynomial.

Corollary 3.2. For every fibered knot $K^{\prime}$ there exist infinitely many non-fibered knots $\left\{K_{n}\right\}_{n \in N}$ each of which has the same Alexander module with $K^{\prime}$. Furthermore, if $K^{\prime}$ is a prime knot, then $K_{n}$ can be taken to be prime.

Proof. Let $K^{\prime}$ be a fibered knot and fix $n^{\prime}>1$. By Theorem 2.3 there exists a knot $K_{1}$ such that

$$
\Delta_{K_{1}}(t)=\Delta_{K}(t), \quad K_{1} \stackrel{n^{\prime}}{\longrightarrow} K^{\prime}, \quad \text { and } g\left(K_{1}\right)>g\left(K^{\prime}\right) .
$$

It follows that $2 g\left(K_{1}\right)>\delta\left(K_{1}\right)$ which implies that $K_{1}$ is non-fibered. Clearly $\alpha\left(K_{1}\right) \geq \alpha\left(K_{1}, K^{\prime}\right) \geq n^{\prime}$. Suppose, inductively, that we have constructed nonfibered knots $K_{1}, \ldots, K_{m}$ such that $\alpha\left(K_{m}\right)>\ldots>\alpha\left(K_{1}\right) \geq n^{\prime}$ and $\Delta_{K_{m}}(t)=$ $\ldots=\Delta_{K_{1}}(t)=\Delta_{K}(t)$. Clearly, $K_{1}, \ldots, K_{m}$ are distinct. Now choose $n>>\alpha\left(K_{m}\right)$ and let $K_{m+1}$ be any knot obtained by applying Theorem 2.3 to this $n$.

By Theorem 1.2(b), the knots $\left\{K_{n}\right\}_{n \in \mathbf{N}}$ can be chosen so that $g\left(K_{n+1}\right)>g\left(K_{n}\right)$. By [Ga1], the 3-manifold $K_{n}(0)$ contains a closed, embedded, orientable, nonseparating surface of genus $g\left(K_{n}\right)$ and contains no such surface of smaller genus. It follows that the manifolds $\left\{K_{n}(0)\right\}_{n \in \mathbf{N}}$ are all distinct. On the other hand, since the Alexander module of $K_{n}(0)$ is the same as that of $K_{n}$, all these 3-manifolds have the same Milnor torsion $([\mathrm{Tu}])$. Thus we obtain the following:

Corollary 3.3. Given a fibered knot $K^{\prime}$ there exist infinitely many non-fibered knots $\left\{K_{n}\right\}_{n \in \mathbf{N}}$, such that the 3-manifolds $\left\{K_{n}(0)\right\}_{n \in \mathbf{N}}$ are all distinct but have the same Milnor torsion with $K^{\prime}(0)$.

\section{Cochran-Melvin invariants of 3-Manifolds}

There exist several constructions of $\mathbf{Z}$-homology 3-spheres that are indistinguishable by Ohtsuki invariants of bounded order. The examples given in $[\mathrm{K}]$ are obtained by Dehn surgery along suitable knots in $S^{3}$. In [CM] Cochran and Melvin generalized Ohtsuki's theory to define finite type invariants for arbitrary 3-manifolds. The knots we construct in this paper, and in particular those in the proof of Theorem 2.3, fit nicely into the theory of $[\mathrm{CM}]$ and lead to a natural extension of the construction of $[\mathrm{K}]$ in this setting. Before we state our results we recall some definitions.

Definition 4.1. A framed link $L$ in a closed, oriented 3-manifold $N$ is called admissible iff we have:

(i) Each component of $L$ is null-homologous in $N$. 
(ii) All the pairwise linking numbers of $L$ in $N$ vanish.

(iii) The framings are \pm 1 with respect to the longitudes given by (i).

Let $N$ be a closed oriented 3-manifold. The set $\mathcal{S}:=\mathcal{S}(N)$ of homeomorphism classes of 3-manifolds that are $H_{1}$-cobordant to $N$ is precisely the set of 3-manifolds obtained by surgery of $N$ along admissible links ([CM]). Let $\mathcal{R}$ be a commutative ring with unit, and let $\mathcal{M}(N)$ be the $\mathcal{R}$-module freely spanned by $\mathcal{S}$. For $M \in \mathcal{S}$ and an admissible link $L \subset M$ define $[M, L] \in \mathcal{M}(N)$ by

$$
[M, L]:=\sum_{L^{\prime} \subset L}(-1)^{\# L^{\prime}} M_{L^{\prime}}
$$

where $L^{\prime}$ ranges over all sublinks of $L$ (including the empty one). Here \# $L^{\prime}$ denotes the number of components of $L^{\prime}$ and $M_{L^{\prime}}$ denotes the 3-manifold obtained from $M$ by surgery along $L^{\prime}$. For $l \geq 0$, let $\mathcal{M}_{l}(N)$ denote the submodule of $\mathcal{M}(N)$ that is freely spanned by all expressions $[M, L]$, where $M \in \mathcal{S}$ and $L$ is an admissible link in $M$ with $\# L \geq l$. Let $\mathcal{H}$ denote the set of $H_{1}$-cobordism classes of closed, oriented 3-manifolds; for $i \in \mathcal{H}$ choose a representative $N_{i}$. Let

$$
\mathcal{M}:=\bigoplus_{i \in \mathcal{H}} \mathcal{M}\left(N_{i}\right) \text { and } \mathcal{M}_{l}:=\bigoplus_{i \in \mathcal{H}} \mathcal{M}_{l}\left(N_{i}\right)
$$

Definition $4.2([\mathrm{CM}])$. A functional $f: \mathcal{M} / \mathcal{M}_{n+1} \longrightarrow \mathcal{R}$ is called an $\mathcal{R}$-valued finite type invariant of order $\leq n$. We will use $\mathcal{F}_{n}$ to denote the space of all such functionals.

One can see that

$$
\mathcal{F}_{n} \cong \bigoplus_{i \in \mathcal{H}} \operatorname{Hom}\left(\mathcal{G}_{n}\left(N_{i}\right), \mathcal{R}\right) \text { where } \mathcal{G}_{n}\left(N_{i}\right):=\mathcal{M}\left(N_{i}\right) / \mathcal{M}_{n+1}\left(N_{i}\right)
$$

Thus, the invariants of finite type of $[\mathrm{CM}]$ are constructed from invariants in each $H_{1}$-cobordism class. Moreover, the invariants of type 0 are exactly the functionals $\mathcal{H} \longrightarrow \mathcal{R}$. In $[\mathrm{CM}]$ it is shown that, for every $n \in \mathbf{N}, \operatorname{Hom}\left(\mathcal{G}_{n}\left(N_{i}\right), \mathcal{R}\right)$ is a finite dimensional non-trivial $\mathcal{R}$-module. To state our results, for a knot $K \subset S^{3}$ and a rational number $s \in \mathbf{Q}$, let $K(s)$ denote the 3 -manifold obtained by $s$-surgery of $S^{3}$ along $K$. Note that $K(s)$ is either a rational homology 3-sphere or a homology $S^{2} \times S^{1}$ manifold.

Proposition 4.3. Suppose that $K, K^{\prime}$ are knots such that $K \stackrel{n}{\longrightarrow} K^{\prime}$, for some $n>0$. Suppose, moreover, that there exists a collection of $n$ ordinary crossings that exhibit $K$ as $n$-adjacent to $K^{\prime}$. Then, for every $s \in \mathbf{Q}$, we have:

$$
f(K(s))=f\left(K^{\prime}(s)\right),
$$

for every $f \in \mathcal{F}_{n-1}$.

Proof. Fix $n>0$ and let $K, K^{\prime}$ be knots such that $K$ admits a collection of ordinary crossings $\mathcal{C}$ that exhibit it as $n$-adjacent to $K^{\prime}$. Let $L \subset S^{3}$ be an $n$-component link consisting of a crossing circle for each of the crossings in $\mathcal{C}$. The crossing change can be achieved by doing surgery of $S^{3}$ along the corresponding crossing circle; the framing of the surgery is +1 or -1 according to whether the crossing is positive or negative. Thus $L$ can be considered as admissible. Since the linking number of 
$K$ with each component of $L$ is zero, each component of $L$ is null-homologous in $S^{3} \backslash K$. Since, for every $s \in \mathbf{Q}$, there is an epimorphism $H_{1}\left(S^{3} \backslash K\right) \longrightarrow H_{1}(K(s))$, it follows that the image of $L$ in $M:=K(s)$ is an admissible link; we will still denote this link by $L$. Let $L^{\prime} \subset L \subset M$ be a non-empty sublink of $L$ in $M$. The 3-manifold $M_{L^{\prime}}$, obtained by surgery of $M$ along $L^{\prime}$, can be alternatively described as follows: First perform surgery of $S^{3}$ along $L^{\prime}$; this gives back $S^{3}$ but it changes $K$ to $K^{\prime}$. Then, perform $s$-Dehn surgery of $S^{3}$ along $K^{\prime}$. From these considerations we conclude that $M_{L^{\prime}}=K^{\prime}(s)$. Thus (2) yields

$$
[M, L]=M-K^{\prime}(s) .
$$

Now let $f \in \mathcal{F}_{n-1}$. Since by definition $f([M, L])=0$, from $(3)$ we obtain $f(M)=$ $f\left(K^{\prime}(s)\right)$ as desired.

Corollary 4.4. Let $n>0$. For every fibered knot $K^{\prime} \subset S^{3}$ there exists a non-fibered knot $K \subset S^{3}$ such that for every $s \in \mathbf{Q}$, we have:

$$
f(K(s))=f\left(K^{\prime}(s)\right),
$$

for every $f \in \mathcal{F}_{n-1}$.

Proof. Given $n$ and $K^{\prime}$ as above, let $K$ be a knot corresponding to $n$ and $K^{\prime}$ in the sense of Theorem 2.3 if $n>1$. Since we do not require $K$ to be prime, we will use Remark 2.4 to conclude that such a $K$ also exists when $n=1$. By the same token, the proof of Theorem 2.3 shows that we can choose $K$ so that it is shown to be $n$-adjacent to $K^{\prime}$ by a collection of ordinary crossings. Thus the corollary follows from Proposition 4.3.

By Gabai's work ([Ga1]), $K(0)$ is irreducible if $K$ is non-trivial and it fibers over $S^{1}$ precisely when $K$ is fibered. Combining these facts with Corollary 4.4 and Theorem 1.2 we obtain Corollary 1.3. Next we give the proof of Corollary 1.4.

Proof of Corollary 1.4. By Theorem 2.3, for every $n \in \mathbf{N}$, there exists a non-trivial knot $K$ that is $n$-adjacent to the trivial knot and has trivial Alexander polynomial. Part (b) of the corollary follows immediately from Proposition 4.3. Corollary 1 of [CGLS] states that if $K$ is not a torus knot, then only for integer slopes $r$, $K(r)$ can have a cyclic fundamental group. Furthermore, there can be at most two such integers, and if there are two they have to be successive. Since $K$ has trivial Alexander polynomial it cannot be a torus knot. Thus part (a) follows immediately from Corollary 1 of [CGLS].

\section{Constructing the Knots $K_{n}$}

In this section we give the proof of Theorem 2.3. First let us explain how Theorem 1.2 follows from Theorem 2.3: Given a fibered knot $K^{\prime}$, for every $n \in \mathbf{N}$, let $K_{n}$ be a non-fibered knot guaranteed by Theorem 2.3. Since $K_{n}$ is $n$-adjacent to $K^{\prime}$, by [KL1], we have $n \leq 6 g\left(K_{n}\right)-3$. Hence $\frac{n+3}{6} \leq g\left(K_{n}\right)$ and parts (a)-(c) of Theorem 1.2 follow. For part (d), we repeat that it follows by Corollary 8.19 of [Ga1].

Before we can proceed with the proof of Theorem 2.3 we need some preparation. First we describe a general construction of a knot $K_{L}^{\bar{q}}$ from a Seifert surface of $K^{\prime}$, an $n$-component string link $L$, and an $n$-tuple of integers $\bar{q}:=\left(q_{1}, \ldots, q_{n}\right)$. Let $S^{\prime} \subset \mathbf{R}^{3}$ be a minimum genus Seifert surface for $K^{\prime}$ and set $g:=\operatorname{genus}\left(S^{\prime}\right)$. 
Suppose that $S^{\prime}$ is isotoped into a disc-band form toward a spine $W_{g}$, which is a bouquet of $2 g$-circles based at a point $p$. Consider a projection $P: \mathbf{R}^{3} \longrightarrow R$ onto a projection plane $R$, so that the restrictions of $P$ to $K^{\prime}$ and $W_{g}$ are both regular. We will identify $W_{g}$ with its diagram under the projection $P$. Let $D \subset R$ be a disc neighborhood of $p$, which contains no crossing points of $W_{g}$. Then, $D$ intersects $W_{g}$ in a bouquet of $4 g$ arcs and the rest of $W_{g}$ consists of $2 g$ arcs outside $D$. We may assume that $S^{\prime}$ is obtained from $W_{g}$ by replacing each of the $\operatorname{arcs}$ outside $D$ by a band. Let $\alpha \subset \partial D$ be a connected subarc containing $W_{g} \cap \partial D$ and set $\alpha^{\prime}:=\partial D \backslash \alpha$. Let

$$
L:\left(I_{n}, \partial I_{n}\right) \longrightarrow\left(\overline{\mathbf{R}^{3} \backslash D \times[0,1]}, \alpha^{\prime}\right)
$$

be an $n$-component string link with components $L_{1}, \ldots, L_{n}$, where $I_{n}$ denotes the disjoint union of $n$-copies of $I:=[0,1]$ and $D=D \times\left\{\frac{1}{2}\right\}$. The end points of $L_{i}$ in $\alpha^{\prime}$ can be joined by a subarc $a_{i}$ in $\alpha^{\prime}$ and we assume that $a_{i} \cap a_{j}=\emptyset$ if $i \neq j$. Furthermore, we will assume that the restriction of $P$ on $L$ is regular and the framing on $L$ defined by parallel copies of $P(L)$ on $R$ is the zero framing. If each $L_{i}$ is a subarc of $\alpha^{\prime}$, we say that the string link $L$ is the trivial string link. A string link $L$ in $\overline{\mathbf{R}^{3} \backslash D \times[0,1]}$ is trivial if it is isotopic to the trivial string link in $\overline{\mathbf{R}^{3} \backslash D \times[0,1]}$ relative to $\partial L$.

We construct a bouquet of $n+2 g$ circles as follows: For $1 \leq i \leq n$, let $p_{i}, p_{i}^{\prime}$ denote the endpoints of $P\left(L_{i}\right)$. Connect $p_{i}, p_{i}^{\prime}$ to $p$ by disjointly embedded arcs $\alpha_{i}, \alpha_{i}^{\prime}$ that lie in $D$ and do not separate any of the arcs in $D \cap W_{g}$. This process yields a bouquet $W_{1}:=W_{1}\left(L, W_{g}\right)$ of $n+2 g$ circles. Note that $W_{1}$ contains a sub-bouquet, say $W_{L}$, whose circles correspond to the components of $L$.

Let $\bar{q}:=\left(q_{1}, \ldots, q_{n}\right)$ be an $n$-tuple of integers. For the circle in $W_{L}$ that corresponds to the component $L_{i}$ of $L$ we add to $W_{1}$ an unlinked and unknotted loop $L_{i}^{\prime}$, which contains $q_{i}$ kinks. This is done in such a way so that the four $\operatorname{arcs}$ of $L_{i}$ and $L_{i}^{\prime}$ in $D$ appear in alternating order. See Figure 1 . This produces a bouquet $W$ of $2(n+g)$ circles such that $D \cap W$ is a bouquet of $4(n+g)$ arcs and there are $2(n+g)$ arcs outside $D$. Now we obtain a surface $S_{L}^{\bar{q}}$ by replacing each of the $\operatorname{arcs}$ outside $D$ by a band, with twists replacing the kinks contained on the arc. Let $K_{L}^{\bar{q}}:=\partial S_{L}^{\bar{q}}$. If there is no danger of confusion we will simply use $K_{L}$ to denote any of the knots $K_{L}^{\bar{q}}$. Next we prove two lemmas needed for the proof of Theorem 2.3.

Lemma 5.1. Let $n>1$. Suppose that the string link $L$ has the following property: Every proper sublink $L^{\prime} \subset L$ can be isotoped, relative to $\partial L^{\prime}$, in the complement of $W_{g}$ in $\overline{\mathbf{R}^{3} \backslash D \times[0,1]}$, to the trivial string link in $\overline{\mathbf{R}^{3} \backslash D \times[0,1]}$. Then, $K_{L}^{\bar{q}}$ is $n$-adjacent to $K^{\prime}$ and it shares a common equivalence class of Seifert matrices with $K^{\prime}$. Thus, in particular, $K_{L}^{\bar{q}}$ and $K^{\prime}$ have the same Alexander module.

Proof. By construction of $S_{L}^{\bar{q}}$, for $1 \leq i \leq n$, the kinks on $L_{i}^{\prime}$ give rise to a generalized crossing, say $C_{i}$, of order $q_{i}$ on $K$. Performing the generalized crossing changes in any non-empty subset of $\left\{C_{1}, C_{2}, \ldots, C_{n}\right\}$ will change $K_{L}^{\bar{q}}$ to $K_{L^{\prime}}$ where $L^{\prime}$ is a proper subset of $L$. By the assumption on $L^{\prime}, K_{L^{\prime}}$ is isotopic to $K^{\prime}$. Thus $K_{L}^{\bar{q}}$ is $n$-adjacent to $K^{\prime}$.

Let $V$ denote the Seifert matrix of $S_{L}$ corresponding to the spine $W$, and $V^{\prime}$ denote the Seifert matrix of $S^{\prime}$ corresponding to the spine $W_{g}$. Since the linking number of $L_{i}$ with each circle in $W_{g}$ is zero, and the linking numbers between $L_{i}$ 


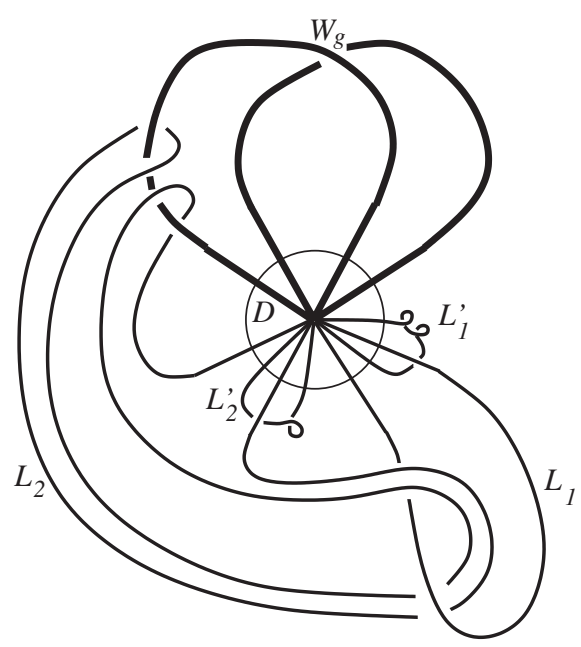

FiguRE 1. An example of a bouquet $W$ and $D \cap W$

and $L_{j}$ are all zero, we see that

$$
V=\left[\begin{array}{cccccc}
V^{\prime} & 0 & 0 & \ldots & 0 & 0 \\
0 & 0 & 0 & \ldots & 0 & 0 \\
0 & 1 & q_{1} & \ldots & 0 & 0 \\
& \ldots & \ldots & \ldots & \ldots & \\
0 & 0 & 0 & \ldots & 0 & 0 \\
0 & 0 & 0 & \ldots & 1 & q_{n}
\end{array}\right] .
$$

Thus, $K_{L}^{\bar{q}}$ and $K^{\prime}$ have the same Alexander module.

For the rest of the section we will assume that $L$ is chosen to satisfy the hypothesis of Lemma 5.1. The next lemma describes the circumstances under which $K_{L}^{\bar{q}}$ is isotopic to $K^{\prime}$.

Lemma 5.2. Let $K^{\prime}$ be a fibered knot. Let $L$ be a string link in $\overline{\mathbf{R}^{3} \backslash D \times[0,1]}$ as in Lemma 5.1. Then, if $K_{L}^{\bar{q}}$ is isotopic to $K^{\prime}, L$ can be isotoped, relative to $\partial L$, in the complement of $W_{g}$ in $\overline{\mathbf{R}^{3} \backslash D \times[0,1]}$, to the trivial string link in $\overline{\mathbf{R}^{3} \backslash D \times[0,1]}$.

Proof. Suppose that $K_{L}^{\bar{q}}$ is isotopic to $K^{\prime}$. For $i=1, \ldots, n$, let $D_{i}$ be a crossing disc corresponding to $C_{i}$ and let $K_{i}:=\partial D_{i}$. We can choose $K_{i}$ to be a small circle linking once around the band of $S_{L}^{\bar{q}}$ corresponding to $L_{i}^{\prime}$. Let $\bar{K}_{L}^{\bar{q}}$ denote the knot obtained from $K_{L}$ by changing all the generalized crossings $C_{1}, \ldots, C_{n}$, simultaneously. One can see that $\bar{K}_{L}$ is obtained from $K^{\prime}$ by $n$ finger moves, one for each component of $L$. More specifically, to obtain $\bar{K}_{L}$, for $i=1, \ldots, n$, one pushes a small part of $K^{\prime}=\partial S^{\prime}$ that contains one endpoint of $L_{i}$, following $L_{i}$ until one gets very close to the other endpoint of $L_{i}$. Then $K_{L}^{\bar{q}}$ is obtained by allowing these fingers to intersect $\bar{K}_{L}$ so as to create the generalized crossings $C_{1}, \ldots, C_{n}$. See Figure 2. It follows that $D_{1}, \ldots, D_{n}$ are also crossing discs for $K^{\prime}$.

By Theorem 3.1 of [KL1], a Seifert surface for $K_{L}^{\bar{q}}$ that is of minimum genus in the complement of $K_{1} \cup \ldots \cup K_{n}$ has to be a minimum genus surface for $K_{L}^{\bar{q}}$. Since we assumed that genus $\left(K_{L}^{\bar{q}}\right)=\operatorname{genus}\left(K^{\prime}\right)=g$, we conclude that $K_{L}^{\bar{q}}$ bounds 

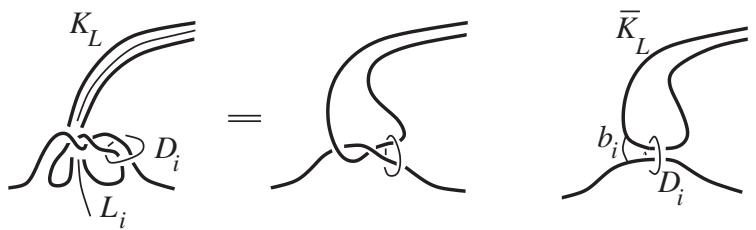

Figure 2. Constructing $K_{L}^{\bar{q}}$ from $\bar{K}_{L}$

a Seifert surface of genus $g$ in the complement of $K_{1} \cup \ldots \cup K_{n}$. Since $\bar{K}_{L}$ is obtained from $K_{L}^{\bar{q}}$ by twisting along $D_{1}, \ldots, D_{n}$, the links $\bar{K}_{L} \cup K_{1} \cup \ldots \cup K_{n}$ and $K_{L}^{\bar{q}} \cup K_{1} \cup \ldots \cup K_{n}$ have homeomorphic complements. We conclude that $\bar{K}_{L}$ bounds a Seifert surface $\Sigma$ of genus $g$ in the complement of $K_{1} \cup \ldots \cup K_{n}$. Since $\Sigma$ is incompressible, by isotopy of $\Sigma$ relative to $\partial \Sigma=\bar{K}_{L}$, we can arrange so that $\Sigma \cap D_{i}$ is a single arc $b_{i}$ properly embedded in $\Sigma$. Each $\operatorname{arc} b_{i}$ is a "short" subarc of $L_{i}$.

Clearly, performing the isotopy from $K^{\prime}$ to $\bar{K}_{L}$ described earlier backwards isotopes the graph $b_{1} \cup \ldots \cup b_{n} \cup \bar{K}_{L}$ onto $L \cup K^{\prime}$. This isotopy brings $\Sigma$ to a minimal genus Seifert surface $\Sigma^{\prime}$ of $K^{\prime}$. The string link $L$ lies on $\Sigma^{\prime}$ as proper arcs. Since $K^{\prime}$ is fibered, it admits a unique minimum genus Seifert surface up to isotopy leaving $K^{\prime}$ fixed pointwise (see, for example, [BZ]). So, $\Sigma^{\prime}$ and $S^{\prime}$ are isotopic relative to $K^{\prime}$. Since $L$ is disjoint from $S^{\prime}$, we may assume that during the isotopy from $\Sigma^{\prime}$ to $S^{\prime}, L$ never touches $S^{\prime}$ except for the last moment when $\Sigma^{\prime}$ and $S^{\prime}$ become identical. The isotopy from $L$ to its image on $S^{\prime}$ are in the complement of $S^{\prime}$ and relative to $K^{\prime}$.

On the other hand, by [K1], each crossing $C_{i}$ must be nugatory. If $C_{i}$ is a nontrivial nugatory crossing, we could have the closure of the corresponding component of $L$ isotoped to a non-trivial summand in the connected sum decomposition of $K^{\prime}$. This contradicts the assumption that each component of $L$ is trivial as a string link. Thus, we may assume that the image of $L$ on $S^{\prime}$ is a collection of inessential proper arcs on $S^{\prime}$. The position of the end points of this collection of inessential proper arcs force them to bound disjoint disks on $S^{\prime}$. We may assume that each of these disks lies in $D$, with one possible exception. For this exceptional disk, the corresponding proper arc in $S^{\prime}$ would run out of $D$, follow the part of $K^{\prime}$ outside of $D$, and come back to $D$. Then the closure of the corresponding component of $L$ would have been isotopic to $K^{\prime}$. This is impossible. Thus the string link $L$ can be isotoped, relative to $\partial L$, in the complement of $W_{g}$ in $\overline{\mathbf{R}^{3} \backslash D \times[0,1]}$, to the trivial string link in $\overline{\mathbf{R}^{3} \backslash D \times[0,1]}$.

Next we turn our attention to the question of whether $K_{L}^{\bar{q}}$ can be chosen to be prime. For this we need the following lemma:

Lemma 5.3. Suppose that $K^{\prime}$ is a prime knot and let $\bar{q}$ be such that $\left|q_{i}\right|>1$, for some $1 \leq i \leq n$. Suppose, moreover, that $g\left(K_{L}^{\bar{q}}\right)>g\left(K^{\prime}\right)$, where $K_{L}^{\bar{q}}$ is a knot associated to $L, \bar{q}$ as above. If $K_{L}^{\bar{q}}$ is a composite knot, then $K^{\prime}$ is a summand of $K_{L}^{\bar{q}}$ and a 2-sphere realizing the corresponding decomposition of $K_{L}^{\bar{q}}$ separates $L$ from $W_{g}$.

Proof. Suppose, without loss of generality, that $\left|q_{1}\right|>1$ and let $K_{1}$ be a crossing link for the generalized crossing $C_{1}$ of $K_{L}^{\bar{q}}$. Suppose that $K_{L}^{\bar{q}}$ has a non-trivial 
connect sum composition $K_{L}^{\bar{q}}=J_{1} \# J_{2}$ and let $T$ be the corresponding followswallow torus. Since $g\left(K_{L}^{\bar{q}}\right)>g\left(K^{\prime}\right)$, by Corollary 4.4 of [ST], $K_{1}$ can be isotoped in the complement of $K_{L}^{q}$ so that it is disjoint from $T$. Let $V$ be the solid torus bounded by $T$; by assumption $J_{1}$ is the core of $V$. Suppose that $K_{1}$ lies outside $V$; the case that $K_{1}$ lies inside $V$ is completely analogous. Then, $C_{1}$ is a crossing on $J_{1}$. The knot obtained from $K_{L}^{\bar{q}}$ by changing $C_{1}$ is of the form $J_{1}^{\prime} \# J_{2}$, where $J_{1}^{\prime}$ is the knot resulting from $J_{1}$. By our assumptions on $L, J_{1}^{\prime} \# J_{2}=K^{\prime}$. Since $K^{\prime}$ is prime it follows that $J_{2}=K^{\prime}$ and $J_{1}^{\prime}$ is the unknot. Thus $K_{L}^{\bar{q}}=J_{1} \# K^{\prime}$. Let $Y$ be a 2-sphere that realizes this connect sum. The surface $S^{\prime}$ of $K^{\prime}$ can be isotoped so that $S^{\prime} \cap Y$ is an arc, say $\gamma$, properly embedded on $D$ such that the points in $\partial \gamma$ leave the arcs $\alpha, \alpha^{\prime} \subset \partial D$ in different components of $S^{3} \backslash Y$. Thus, in particular, $Y$ separates $\partial L$ from $W_{g}$. Recall that $K_{L}^{\bar{q}}$ is the boundary of a surface obtained from $S^{\prime} \cup L$ by replacing each component of $L$ by an appropriate band. Now, since $K_{L}^{\bar{q}} \cap Y=\partial \gamma$, it follows that $Y$ separates $L$ from $W_{g}$.

We can now finish the proof of Theorem 2.3:

Proof of Theorem 2.3. Let $n>1$. Let $S^{\prime}$ be a minimum genus Seifert surface for $K^{\prime}$ and a projection of it on $R$ as fixed earlier. Choose $L:\left(I_{n}, \partial I_{n}\right) \longrightarrow\left(\overline{\mathbf{R}^{3} \backslash D}, \alpha^{\prime}\right)$ such that:

(i) Every proper sublink $L^{\prime} \subset L$ can be isotoped, relatively $\partial L^{\prime}$, in the complement of $W_{g} \cup K^{\prime}$ so that it is properly embedded in $D$.

(ii) $L$ is not trivial.

(iii) There is no 2-sphere that intersects $K^{\prime}$ at exactly two points and separates $L$ from $W_{g}$.

Let $\bar{q}$ be such that $\left|q_{i}\right|>1$, for some $1 \leq i \leq n$. We claim that the knot $K:=K_{L}^{\bar{q}}$ has properties (a)-(d). Properties (a), (b), follow from (i) and Lemma 5.1. By (ii) and Lemma $5.2, K$ is not isotopic to $K^{\prime}$; thus by Theorem $2.2, g(K)>g\left(K^{\prime}\right)$. Now part (d) follows immediately from Lemma 5.3.

\section{Appendix A. ObstruCting Symplectic struCtures}

In recent years knots that look fibered to the Alexander polynomial have received particular attention in symplectic geometry. For example, a problem of current interest is when a 4-manifold of the form $S^{1} \times M$, where $M$ is a 3-manifold, admits a symplectic structure. It is known that if $K$ is fibered, then $S^{1} \times K(0)$ admits a symplectic structure and it has been conjectured that the converse is true (see [Kr1] and the references therein). It is known that the Alexander polynomial of a knot $K$ obstructs to the existence of symplectic structures on $S^{1} \times K(0)$. More specifically, it is known that if $S^{1} \times K(0)$ admits a symplectic structure, then $\Delta_{K}(t)$ is monic. Furthermore, by a result of Kronheimer ([Kr2]), if $g(K)>1$, we must have $m(K)=0$. Combining this with Proposition 3.1, it follows that $\alpha(K)$ is a secondary obstruction to the existence of symplectic structures on $S^{1} \times K(0)$ :

Theorem A.1. Let $K \in \mathcal{K}_{1}$. If $S^{1} \times K(0)$ admits a symplectic structure, then $\alpha(K)=0$.

Proof. If $g(K)>1$ the conclusion follows immediately from the aforementioned result of Kronheimer and Proposition 3.1 (b). Suppose that $g(K)=1$ and let $K^{\prime}$ be a fibered knot such that $\Delta_{K}(t)=\Delta_{K^{\prime}}(t)$ and $K \stackrel{n}{\longrightarrow} K^{\prime}$, for some $n>1$. By Theorem 2.2, $g(K)>g\left(K^{\prime}\right)$ and, since $g(K)=1, K^{\prime}$ is the trivial knot. Hence 
$\Delta_{K}(t)$ is trivial. By Theorem 5.1 of $[\mathrm{KL}]$, the only genus one knots that are at least 2-adjacent to the unknot are 2-bridge knots. But the only 2-bridge knot with trivial Alexander polynomial is the unknot. Thus $K=K^{\prime}$, and by definition, $\alpha(K)=0$.

Let $K_{n}, K^{\prime}$ be knots as in Corollary 3.2. Since $K^{\prime}$ is fibered, $S^{1} \times K^{\prime}(0)$ is symplectic. Since, by construction, $\alpha\left(K_{n}\right)>0$, Theorem A.1 implies that $S^{1} \times K_{n}(0)$ doesn't admit symplectic structures. Thus we have examples of non-symplectic 4-manifolds that are not distinguished from symplectic ones by the information contained in the Alexander polynomial.

\section{ACKNOWLEDGMENT}

Effie Kalfagianni thanks the Institute for Advanced Study for their hospitality while part of the research described in this paper was completed and for their partial support through a research grant. She also acknowledges the partial support of the NSF through grants DMS-0306995 and FRG/DMS-0456155. Xiao-Song Lin acknowledges the partial support of the NSF through grants DMS-0404511 and FRG/DMS-0456217.

\section{REFERENCES}

[BZ] G. Burde and H. Zieschang, Knots. de Gruyter Studies in Mathematics, 5. Walter de Gruyter \& Co., Berlin, 1985. MR808776 (87b:57004)

[Ch] Cha, Jae Choon, Fibred knots and twisted Alexander invariants, Trans. Amer. Math. Soc. 355 (2003), no. 10, 4187-4200. MR1990582 (2004e:57008)

[C] T. Cochran, Noncommutative knot theory, Algebr. Geom. Topol. 4 (2004), 347-398. MR2077670 (2005k:57023)

[CM] T. Cochran and P. Melvin, Finite type invariants of 3-manifolds, Invent. Math. 140 (2000), no. 1, 45-100. MR1779798 (2002a:57015)

[CGLS] M. Culler, C. Gordon, J. Luecke, and P. Shalen, Dehn surgery on knots, Ann. of Math. (2) 125 (1987), no. 2, 237-300. MR881270 (88a:57026)

[Ga1] D. Gabai, Foliations and the topology of 3-manifolds III, J. Diff. Geom. vol 26 (1987), 479-536. MR910018 (89a:57014b)

[FKi] S. Friedl and T. Kim, Thurston norm, fibered manifolds and twisted Alexander polynomials, Topology 45 (2006), no. 6, 929-953. MR2263219

[Ga2] D. Gabai, Detecting fibred links in $S^{3}$, Comment. Math. Helv. 61 (1986), no. 4, 519-555. MR870705 (88c:57009)

[GKM] H. Goda, T. Kitano and T. Morifuji, Reidemeister torsion, twisted Alexander polynomial and fibered knots, Comment. Math. Helv. 80 (2005), no. 1, 51-61. MR2130565 (2005m:57008)

[K] E. Kalfagianni, Homology spheres with the same finite type invariants of bounded orders, Math. Res. Lett. 4 (1997), no. 2-3, 341-347. MR1453064 (98h:57033)

[K1] E. Kalfagianni, Crossing changes of fibered knots. math.GT/0610440

[KL] E. Kalfagianni and X.-S. Lin, Knot adjacency and satellites, Topology and Applications, 138 (2004), 207-217. MR2035481 (2005e:57019)

[KL1] E. Kalfagianni and X.-S. Lin, Knot adjacency, genus and essential tori, Pacific Jour. of Mathematics, Vol. 228, no. 2, 251-276 (2006). MR2274520 (2007k:57010)

[Kr1] P. B. Kronheimer, Embedded surfaces and gauge theory in three and four dimensions, Surveys in differential geometry, Vol. III (Cambridge, MA, 1996), 243-298, Int. Press, Boston, MA, 1998. MR1677890 (2000a:57086)

[Kr2] P. B. Kronheimer, Minimal genus in $S^{1} \times M^{3}$, Invent. Math. 135 (1999), no. 1, 45-61. MR1664695 (2000c:57071)

[Mo] H. R. Morton, Fibred knots with a given Alexander polynomial. Knots, braids and singularities , 205-222, Monogr. Enseign. Math., 31, Enseignement Math., Geneva, 1983. MR728587 (85j:57006) 
[O] T. Ohtsuki, Finite type invariants of integral homology 3-spheres, J. Knot Theory Ramifications 5 (1996), no. 1, 101-115. MR1373813 (97i:57019)

[ST] M. Scharlemann and A. Thompson, Unknotting number, genus, and companion tori, Math. Ann. 280 (1988), no. 2, 191-205. MR929535 (89d:57008)

[Tu] V. Turaev, Torsions of 3-dimensional manifolds. Progress in Mathematics, 208. Birkhäuser Verlag, Basel, 2002. MR1958479 (2003m:57028)

Department of Mathematics, Michigan State University, East Lansing, Michigan 48824

E-mail address: kalfagia@math.msu.edu

Department of Mathematics, University of California, Riverside, California 92521 